\title{
Exploring the Influence of different Albumin-Binders on Molecular Imaging Probe Distribution
}

\author{
Carsten Höltke ${ }^{1, *}$, Martin Grewer ${ }^{1}$, Miriam Stölting ${ }^{1}$, Christiane Geyer ${ }^{1}$, Moritz \\ Wildgruber ${ }^{1,2}$ and Anne Helfen ${ }^{1}$ \\ 1) Clinic for Radiology, University Hospital Münster, Münster, Germany; 2) Department of \\ Radiology, University Hospital, LMU Munich, Munich, Germany.
}

\section{Supporting Information}

Synthesis of probes. Fatty acid derived probe 3: Boc-L-Lys(Palm)-OH (249mg, $0.515 \mathrm{mmol}$, Iris Biotech $\mathrm{GmbH}$, Marktredwitz, Germany) and TBTU (222mg, 0.690mmol) were dissolved in DMF $(25 \mathrm{~mL})$. After addition of DIPEA $(200 \mu \mathrm{L}, 1.15 \mathrm{mmol})$ the solution was stirred for $30 \mathrm{~min}$. Compound 1 (363mg, $0.514 \mathrm{mmol}$ ) was dissolved in DMF $(20 \mathrm{~mL})$ and added to the former solution, the mixture was stirred overnight at rt. Water and ethyl acetate were added and the mixture acidified with diluted hydrochloric acid. The phases were separated and the aqueous phase was extracted with ethyl acetate $(3 \mathrm{x})$. The combined organic extracts were washed with brine and dried $\left(\mathrm{MgSO}_{4}\right)$. After removal of the solvent the residue was purified by column chromatography $(\mathrm{EtOAc}+5 \% \mathrm{MeOH})$. The pure Boc-protected compound (MS analysis by MicroTof/ESI: $\mathrm{m} / \mathrm{z}=1156.6267, \mathrm{M}+\mathrm{Na}^{+}$; calc.: C62H91N3O16Na+ $=1156.6292$ ) was taken up in $5 \mathrm{~mL}$ of dioxane and treated with $4 \mathrm{M} \mathrm{HCl}$ in dioxane $(15 \mathrm{~mL})$ for $2 \mathrm{~h}$ at room temperature. The solvent was evaporated and the residue lyophilized yielding $450 \mathrm{mg}(348 \mu \mathrm{mol})$ of the fatty acid amine as hydrochloride (MS analysis by MicroTof/ESI: $\mathrm{m} / \mathrm{z}=1034.5930[\mathrm{M}+\mathrm{H}]^{+}$; calc.: C57H84N3O14 $\left.{ }^{+}=1034.5948\right)$. Conjugation of the Cy 5.5 dye was accomplished by dissolving $1.2 \mathrm{mg}$ of dye NHS-ester $(1.0 \mu \mathrm{mol})$ in $300 \mu \mathrm{L}$ of DMSO and adding a solution of $2.5 \mathrm{mg} 3.1$ $(2.0 \mu \mathrm{mol})$ in DMF containing $50 \mu \mathrm{L}(250 \mu \mathrm{mol})$ DIPEA. Shaking the mixture overnight in the dark and subsequent HPLC purification (gradient from $80 \%$ water to $20 \%$ water in $25 \mathrm{~min}$, semipreparative C18 column, Nucleosil, $\varnothing=8 \mathrm{~mm}$ ) yielded the probe 3 in $>95 \%$ purity. MS analysis (Orbitrap/ESI): m/z = 643.24106 [M-3H] ${ }^{3-}$ (calc.: C98H122N5O27S4- = 643.24194), 965.36579 [M-2H] $]^{2-}$ (calc.: C98H123N5O27S42- = 965.36655), $976.35722[\mathrm{M}-3 \mathrm{H}+\mathrm{Na}]^{2-}$ (calc.: $\left.\mathrm{C} 98 \mathrm{H} 122 \mathrm{~N} 5 \mathrm{O} 27 \mathrm{~S} 4 \mathrm{Na}^{2-}=976.35761\right)$. The concentration of the reconstituted (purified water) probe solution was measured by determining the absorption of serially diluted aliquots and applying Lambert-Beer's law, using the excitation coefficient of Cy 5.5 dye given in the literature.

p-lodophenylbutyric acid derivative 4: 4-(p-lodophenyl)butyric acid $(871 \mathrm{mg}, 3.0 \mathrm{mmol})$ was dissolved in $10 \mathrm{~mL}$ of DMF and treated with EDC (770mg, $4.0 \mathrm{mmol}), \mathrm{HOBt}(615 \mathrm{mg}, 4.0 \mathrm{mmol})$ and DIPEA $(850 \mu \mathrm{L}, 5.0 \mathrm{mmol})$ for 30 minutes at $0^{\circ} \mathrm{C}$. Boc-L-Lys-OMe acetate $(781 \mathrm{mg}$, 
3.0mmol) and DIPEA (850 $\mathrm{L}, 5.0 \mathrm{mmol})$ dissolved in $10 \mathrm{~mL}$ DMF were added and the mixture was stirred overnight at rt. Water was added and the mixture acidified with acetic acid $(\mathrm{pH} 4)$. Extraction with $\mathrm{CH}_{2} \mathrm{Cl}_{2}(3 \mathrm{x})$, washing with brine and drying with $\mathrm{MgSO}_{4}$ yielded the crude methyl ester, tert-butyl (S)-(8-(4-(4-iodophenyl)butanamido)-2,2-dimethyl-3-oxooctan-4$\mathrm{yl}$ )carbamate, which was purified by column chromatography (cyclohexane/ethyl acetate, 2:1 $\rightarrow 1: 1$ ). Yield: $702 \mathrm{mg}, 1.3 \mathrm{mmol}$. Saponification of the methyl ester with $\mathrm{LiOH}(160 \mathrm{mg}, 6.5 \mathrm{mmol})$ in $10 \mathrm{~mL} \mathrm{MeOH} / \mathrm{THF} / \mathrm{H}_{2} \mathrm{O}$ (3:1:1) overnight after aqueous workup yielded the carboxylic acid $4.1(620 \mathrm{mg}, 1.2 \mathrm{mmol})$ as a clear viscous oil. Coupling to 1 : The carboxylic acid $4.1(260 \mathrm{mg}$, $0.5 \mathrm{mmol}), 1335 \mathrm{mg}(0.5 \mathrm{mmol})$, TBTU $(320 \mathrm{mg}, 1.0 \mathrm{mmol})$ and $140 \mathrm{mg} \mathrm{HOBt}(0.9 \mathrm{mmol})$ were dissolved in $10 \mathrm{~mL}$ DMF at it and treated with $500 \mu \mathrm{L}$ DIPEA overnight. After aqueous workup and column chromatography (ethyl acetate/methanol 19:1) the product was directly treated with $4 \mathrm{~N} \mathrm{HCl}$ in dioxane overnight to remove the Boc protection group. Evaporation of the solvent gave the desired product as the hydrochloride $(4.2)$ in $62 \%$ yield $(340 \mathrm{mg}, 0.31 \mathrm{mmol})$. MS analysis by MALDI-TOF/ESI: $\mathrm{m} / \mathrm{z}=1068.29[\mathrm{M}+\mathrm{H}]^{+}$, calc.: C51H63IN3O14 ${ }^{+}=1068.33$; $\mathrm{m} / \mathrm{z}=1090.28[\mathrm{M}+\mathrm{Na}]^{+}$, calc.: C51H62IN3014Na $=1090.32 ; \mathrm{m} / \mathrm{z}=1106.26[\mathrm{M}+\mathrm{K}]^{+}$, calc.: $\left.\mathrm{C} 51 \mathrm{H} 62 \mathrm{~N}_{3} \mathrm{O} 14 \mathrm{~K}^{+}=1106.29\right)$. Conjugation of the Cy 5.5 dye was accomplished by dissolving $1.2 \mathrm{mg}$ of dye NHS-ester $(1.0 \mu \mathrm{mol})$ in $300 \mu \mathrm{L}$ of DMSO and adding a solution of $2.2 \mathrm{mg} 4.2$ hydrochloride $(2.0 \mu \mathrm{mol})$ in DMF containing $50 \mu \mathrm{L}(250 \mu \mathrm{mol})$ DIPEA. Shaking the mixture overnight in the dark and subsequent HPLC purification (gradient from $80 \%$ water to $20 \%$ water in $25 \mathrm{~min}$, semipreparative column) yielded the probe $\mathbf{4}$ in $>98 \%$ purity. MS analysis by MALDITOF/ESI: $\mathrm{m} / \mathrm{z}=982.23826[\mathrm{M}-2 \mathrm{H}]^{2-}$, calc.: C92H102IN5O27S42- $=982.23662 ; \mathrm{m} / \mathrm{z}=$ 1965.48283 [M-H]', calc.: C92H103IN5O27S4- = 1965.48051). The concentration of the reconstituted (purified water) probe solution was measured by determining the absorption of serially diluted aliquots and applying Lambert-Beer's law, using the excitation coefficient of Cy 5.5 dye given in the literature. 
A

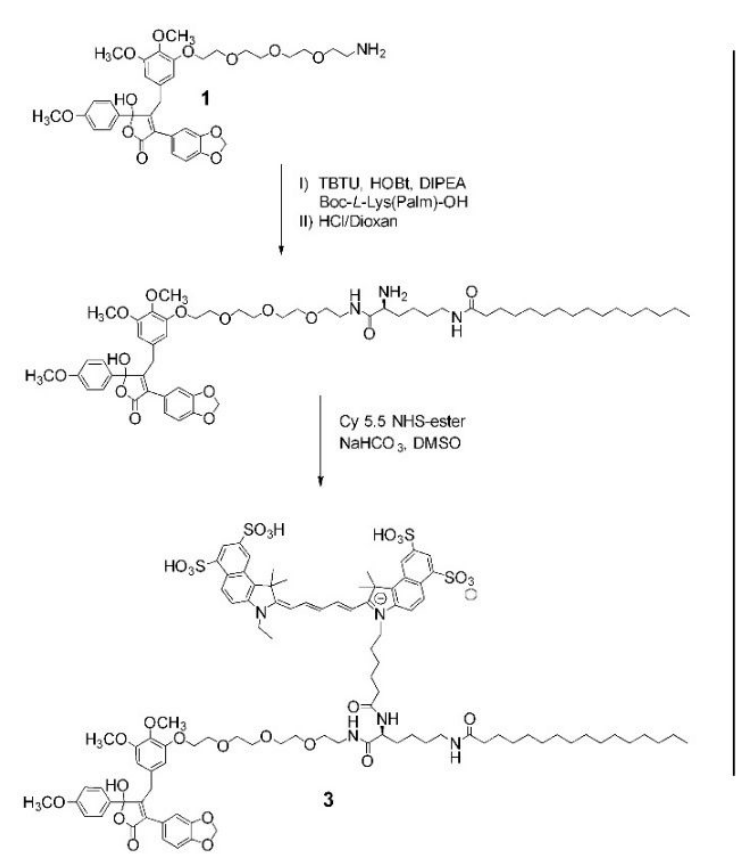

B

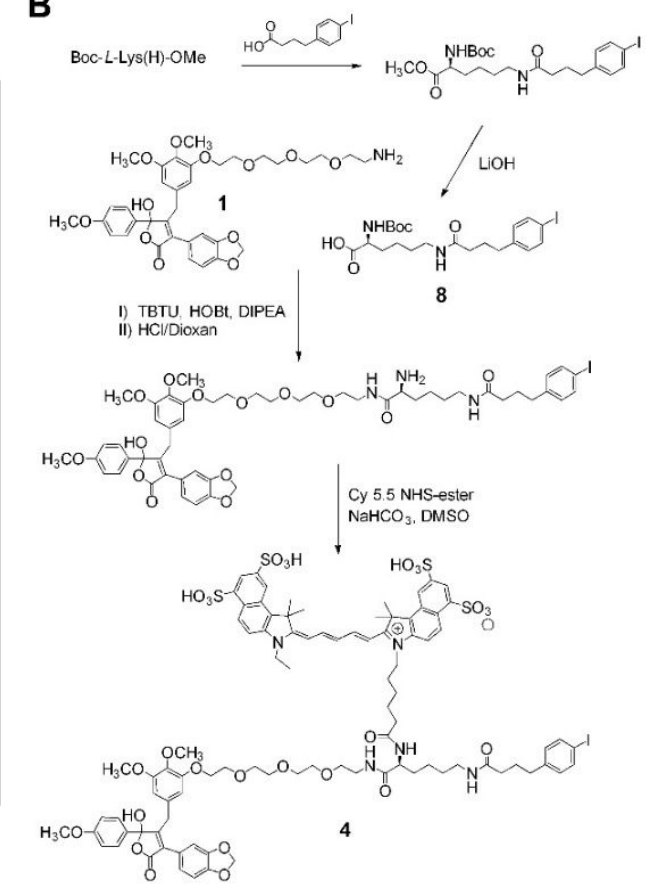

Scheme S1. Synthetic routes towards the fatty acid derived probe $3(\mathbf{A})$ and the $p$-iodophenyl butyric acid derived probe 4 (B). A. Compound 1 is coupled to Boc-L-Lys(Palm)-OH by HBTU/HOBt treatment. After deprotection of the Boc-protecting group with $\mathrm{HCl}$ in dioxane, the dye is coupled in bicarbonate buffer to yield 3. B. Boc-L-Lys $(\mathrm{H})-\mathrm{OMe}$ is coupled to $p$-iodophenyl butyric acid by HBTU/HOBt treatment. After saponification of the methyl ester by LiOH, the ligand $\mathbf{1}$ is attached using similar conditions as in $\mathbf{A}$. After deprotection of the Boc-protecting group with $\mathrm{HCl}$ in dioxane, the dye is coupled in bicarbonate buffer to yield 4 .
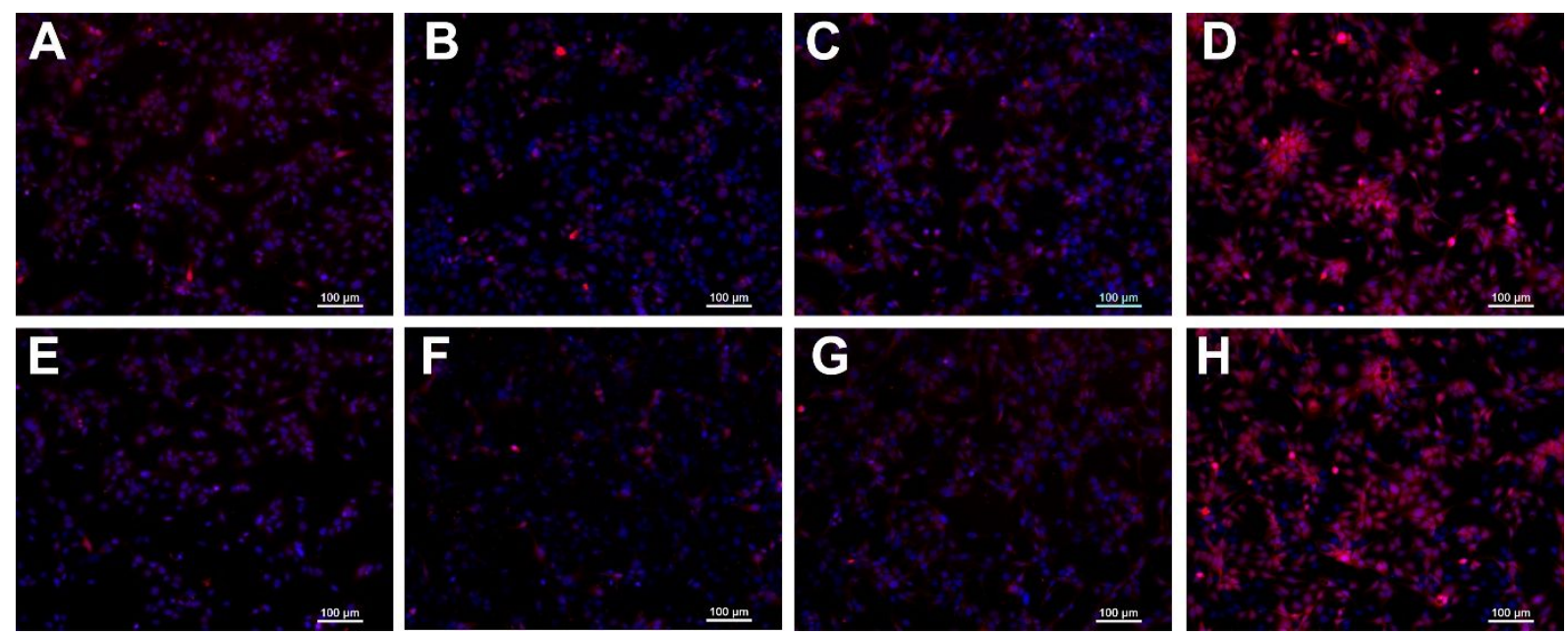

Figure S1. Cell binding (A-D) and competition experiments (E-H) with all four probes on $E T_{A} R$-negative $B T-20$ adenocarcinoma cells show the degree of unspecific binding (exposure time $30 \mathrm{sec}$ ). Compounds $\mathbf{5}, \mathbf{2}$ and $\mathbf{3}$ show a low unspecific staining of the cells $(\mathbf{A}, \mathbf{B}, \mathbf{C}$ and $\mathbf{E}, \mathbf{F}, \mathbf{G})$, while compound $\mathbf{4}$ shows a strong staining of these cells, indicating a high degree of unspecific binding $(\mathbf{D}, \mathbf{H})$. The excess of $E T_{A} R$ antagonist (atrasentan) is not capable of reducing the degree of staining. 
A

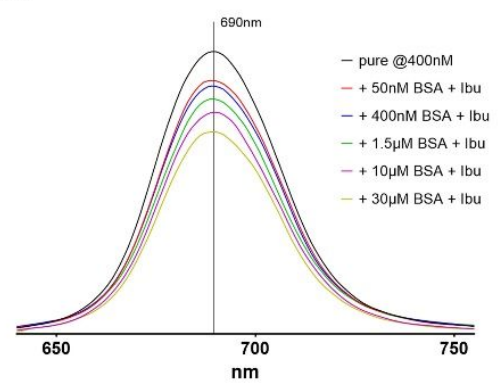

C

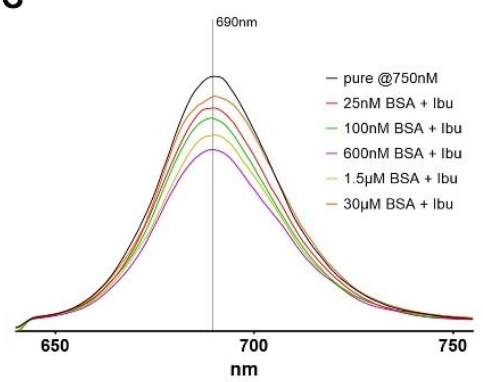

B

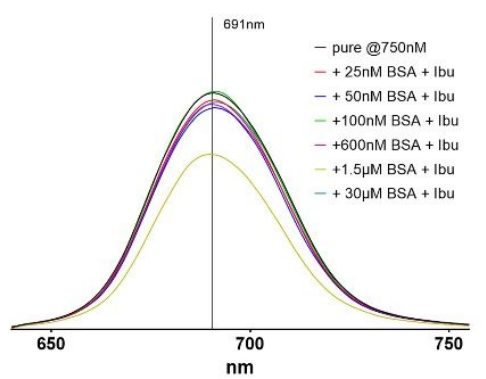

D

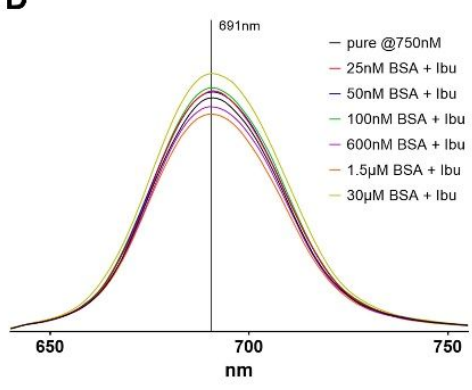

Figure S2. Effect of ibuprofen on the emission spectra of the developed probes (compare to figure $\mathbf{4}$ in the main text, A. Compound 5, B. Compound 2, C, Compound 3, D. Compound 4). The intense reduction in emission intensity is alleviated. Also, the red-shift of the emission maximum is suppressed (conc. of ibuprofen $=486 \mu \mathrm{M}$ in $\mathbf{A} ; 1.86 \mathrm{mM}$ in $\mathbf{B}, \mathbf{C}$ and $\mathbf{D}$ ).

A

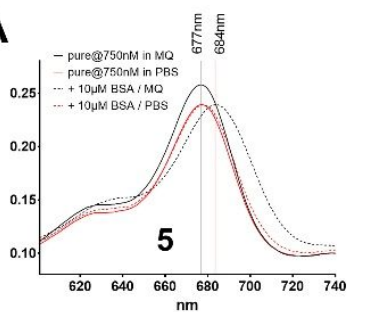

B

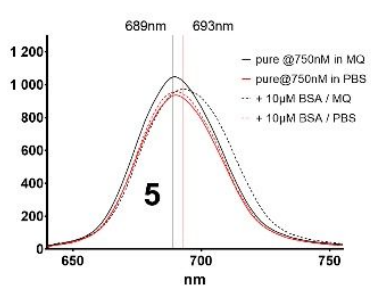

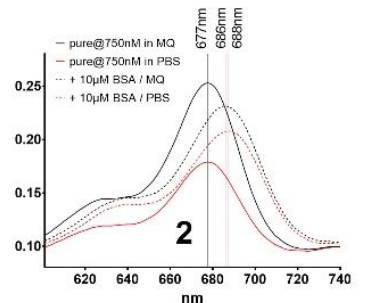

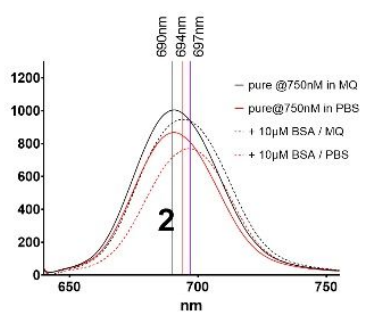

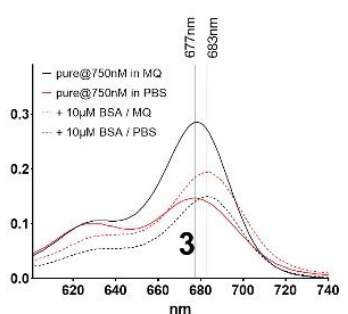
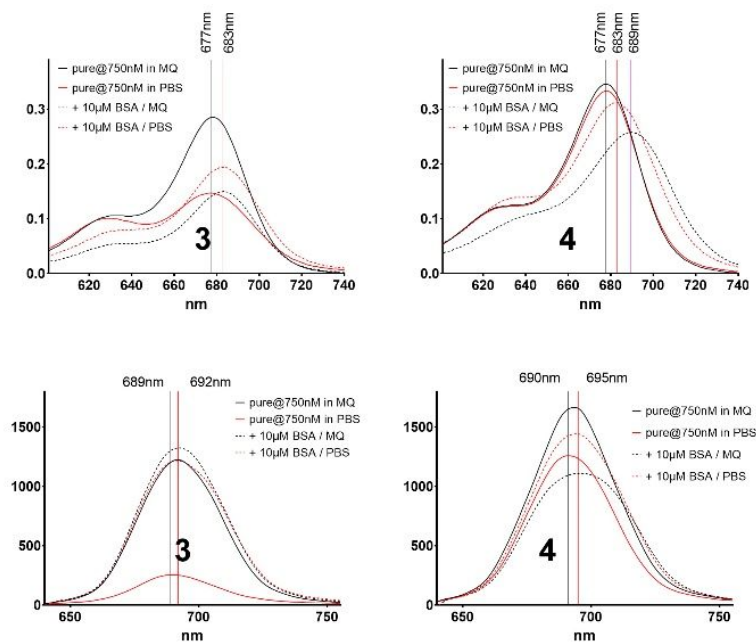

Figure S3. Investigation of solvent-related effects on the absorption (A) and emission (B) characteristics of the developed probes. Probe $\mathbf{5}$ exhibits the lowest effects, both for absorption and emission properties. Especially probes $\mathbf{2}$ and $\mathbf{3}$ show a marked reduction in absorption peak intensity in PBS compared to purified water. This reduction was in part restored when serum albumin was added. Compound $\mathbf{3}$ also shows a strong reduction of emission intensity in PBS, which could completely be restored with the addition of albumin. In some cases, a difference in red-shift extent after serum albumin addition is observed. 
A
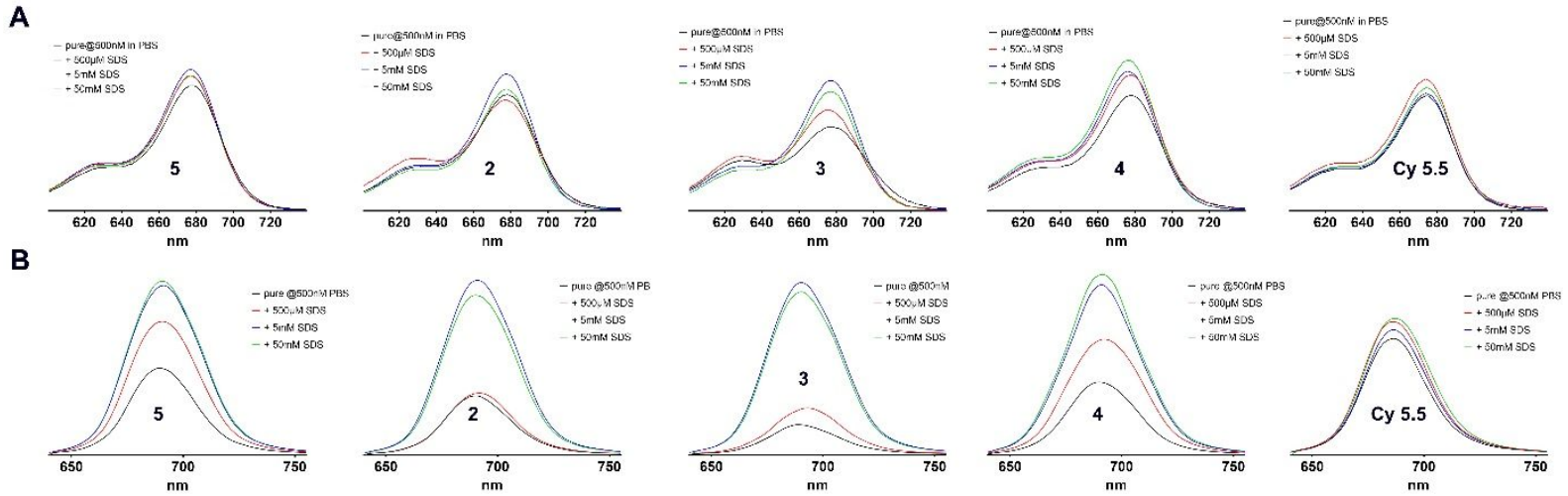

Figure S4. Effect of surfactants (SDS) on the absorption (A) and emission (B) characteristics of the probes 2-5 and the free dye Cy 5.5 in PBS. The absorption spectra of all compounds are only marginally affected by the addition of SDS (A), only probe 3 shows a slight intensity elevation. The emission spectra of the probes are more intensely affected by SDS (B). Compounds 2 and $\mathbf{3}$ show a highly elevated emission peak when SDS concentrations reach the critical micelle concentration (CMC). Probes $\mathbf{4}$ and $\mathbf{5}$ also show an enhancement of emission intensity, but this effect does not seem to be dependent on micelle formation. A red-shift of the absorption or emission maxima is not observed. The pure dye alone does not show any sign of interaction with SDS.
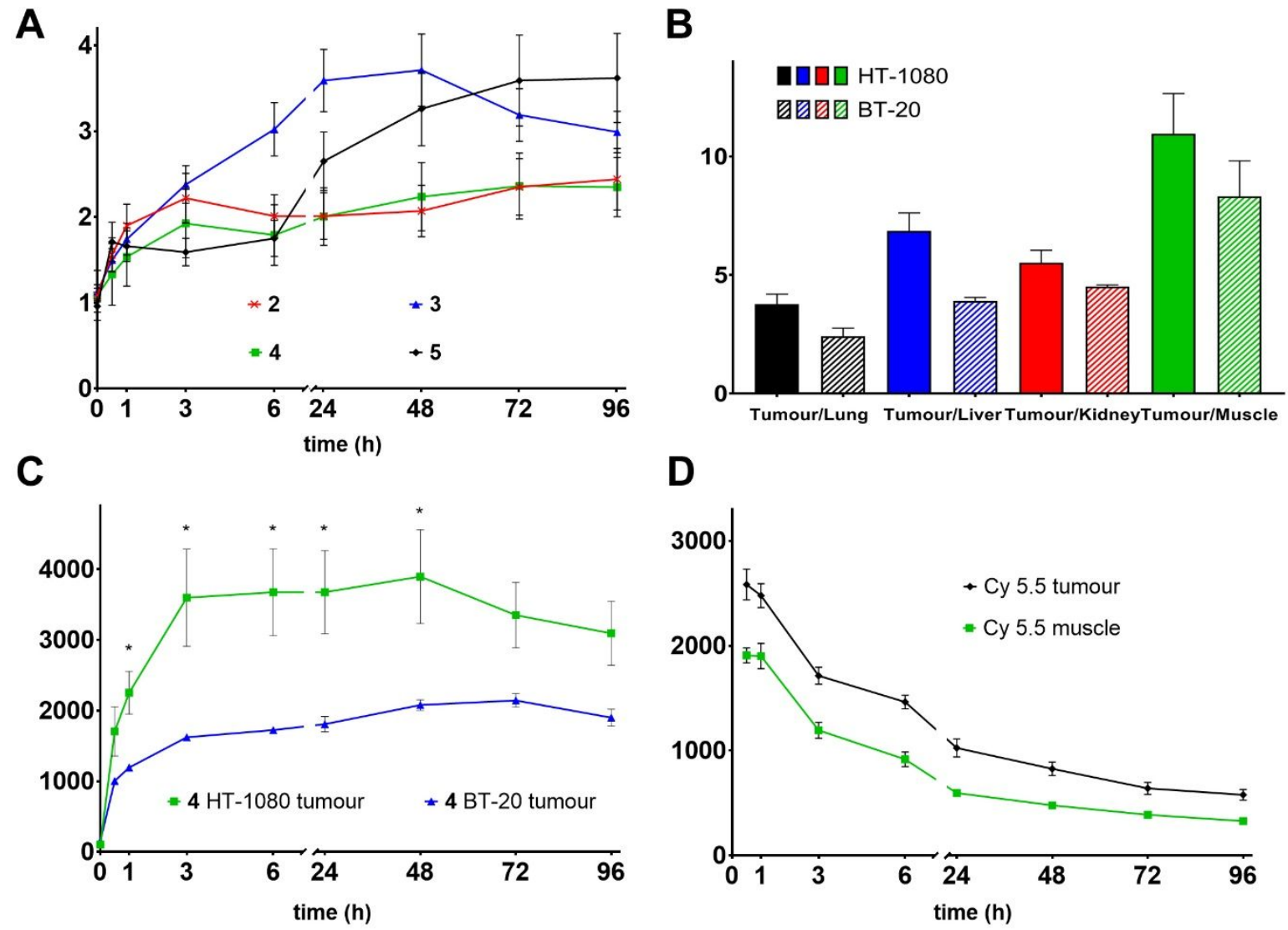

Figure S5. Additional data from in vivo measurements. A: In vivo tumour-to-muscle ratios as derived from FRI measurements (ratios of tumour SI / muscle SI) of HT-1080 tumours implanted in the breast of nude mice (colour coding: black, compound $\mathbf{5}$, red, compound $\mathbf{2}$, blue, compound $\mathbf{3}$, green, compound $\mathbf{4}$ ). The unmodified compound 5 shows rather low values at early time points after bolus application but a marked increase after 24 hours. Compound 3 shows the highest values from 3 hours until $48 \mathrm{~h}$. Compounds 2 and 4 show rather low values. B: Ex vivo tumour-to-organ ratios @96 h pi as derived from FRI measurements (ratios of tumour SI / organ SI) of HT1080 (solid colour) and BT-20 tumours (striped) implanted in the breast of nude mice (colour coding: black, tumour/lung ratio; blue, tumour/liver ratio; red, tumour/kidney ratio; green, tumour/muscle ratio). The experiments 
were performed with compound 4. Data is presented as mean \pm SEM of $n=2-6$ experiments. $\mathbf{C}$ : In vivo accumulation of compound 4 in $E_{A} R$-positive $H T-1080$ and $E T_{A} R$-negative $B T-20$ tumours, revealing a significantly lower signal intensity in control tumours from 1-48 hours. D: In vivo accumulation of pure Cy 5.5 dye in HT-1080 tumours compared to muscle tissue. The time course shows a distinct contrast to that of targeted compounds (compare to figure 5B).
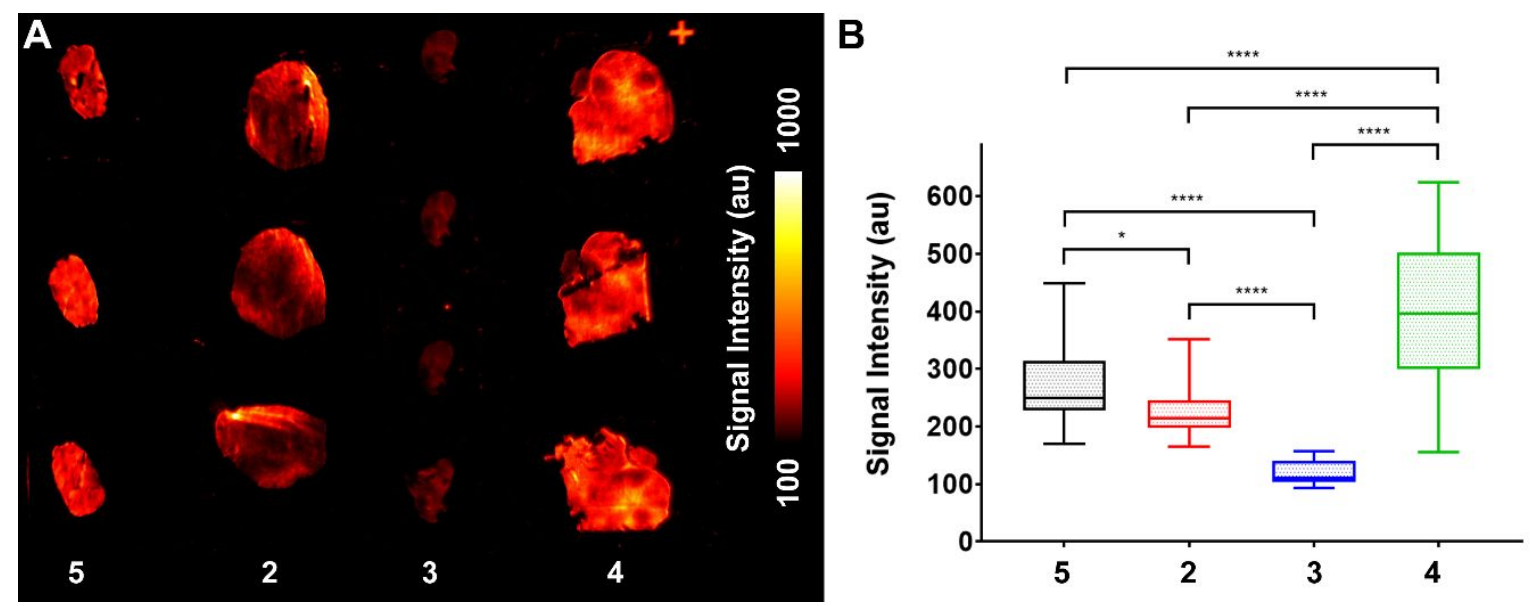

Figure S6. Additional data from ex vivo measurements. A: Examples of tumour sections from the four different probes as imaged by FRI. The fatty acid derived compound $\mathbf{3}$ shows the weakest fluorescence, while the $p$ iodophenyl derived probe 4 is brightest. Analysis of the tumour fluorescence shows significant differences between the probes (compare to figure $\mathbf{5 C}$ ).

Table S1. Table summarizing the p-values of unpaired t-tests performed on the in vivo fluorescence signal intensities of the tumor area at the indicated time-points (FRI measurements, $n=5-8$, compare to figure 5B).

\begin{tabular}{|c|c|c|c|c|c|c|}
\hline & 5 vs. 2 & 5 vs. 3 & 5 vs. 4 & 2 vs. 3 & 2 vs. 4 & 3 vs. 4 \\
\hline $30 \mathrm{~min}$ & $<0.001$ & $<0.0001$ & ns & ns & ns & $<0.05$ \\
\hline $1 \mathrm{~h}$ & $<0.0001$ & $<0.0001$ & ns & ns & $<0.01$ & $<0.001$ \\
\hline $3 \mathrm{~h}$ & ns & $<0.01$ & ns & ns & $<0.01$ & $<0.001$ \\
\hline $6 \mathrm{~h}$ & $\mathrm{~ns}$ & $<0.01$ & ns & ns & $<0.01$ & $<0.001$ \\
\hline $24 \mathrm{~h}$ & ns & ns & ns & ns & $<0.01$ & $<0.01$ \\
\hline $48 h$ & ns & ns & $<0.01$ & ns & $<0.01$ & $<0.001$ \\
\hline $72 \mathrm{~h}$ & ns & ns & $<0.01$ & ns & $<0.01$ & $<0.001$ \\
\hline $96 \mathrm{~h}$ & ns & ns & $<0.01$ & ns & $<0.01$ & $<0.001$ \\
\hline
\end{tabular}

